\title{
Management of melanoma brain metastases
}

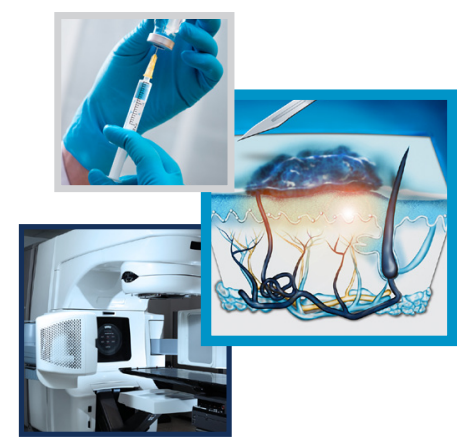

Mary Frances McAleer', Dae W Kim², Van A Trinh ${ }^{3}$ \& Wen-Jen Hwu ${ }^{*, 2}$

\section{Practice points}

- Development of brain metastases (BMs) is a common and devastating event in the course of advanced melanoma.

- Management of melanoma BMs requires comprehensive evaluation of patient's performance status, the number, size and location of $\mathrm{BMs}$, the extent of extracranial disease and BRAF mutation status.

- Surgical resection of BMs improves local disease control, enhances functional independence and may prolong overall survival in patients with good performance status, limited or no extracranial disease, and BMs amenable to safe and complete resection.

- Stereotactic radiosurgery has largely replaced whole brain radiation therapy (WBRT) in patients who are not surgical candidates, with limited BMs, or BMs in inaccessible or eloquent areas of the brain given the relative radioresistance of melanoma to the more toxic WBRT.

- Traditional cytotoxic agents, such as temozolomide or fotemustine, with or without WBRT, have marginal impact on progression-free survival and overall survival in patients with melanoma BMs.

- Novel immunotherapies, such as ipilimumab, and targeted agents, such as vemurafenib and dabrafenib, produce meaningful intracranial response and expand the systemic treatment options for patients with melanoma BMs.

Relapses in the brain remain a major obstacle to cure in many patients with advanced melanoma. At present, the management of melanoma brain metastases continues to rely heavily on surgical and radiotherapeutic interventions, which have become safer and more effective with modern imaging, surgery and radiation technologies. Additionally, novel targeted and immunotherapeutic agents, shown to generate meaningful intracranial response and survival benefit in patients with melanoma brain metastases when compared with historical controls, expand systemic treatment options for this subset of patients. These systemic therapies become particularly important when intracranial disease burden precludes neuro- or radio-surgery. Considerable multidisciplinary research effort is ongoing to improve outcomes for melanoma patients with brain metastases, a key challenge in the management of advanced melanoma.
'Department of Radiation Oncology, The University of Texas MD Anderson Cancer Center, 1515 Holcombe Blvd, Houston, TX 77030 , USA

2Department of Melanoma Medical Oncology, The University of Texas MD Anderson Cancer Center, 1515 Holcombe Blvd, Houston, TX 77030, USA

${ }^{3}$ Clinical Pharmacy Specialist, Pharmacy Clinical Programs, The University of Texas MD Anderson Cancer Center, 1515 Holcombe Blvd, Houston, TX77030, USA

*Author for correspondence: wenjhwu@mdanderson.org
Future $\mathrm{fSS}$ 


\section{KEYWORDS}

- advanced melanoma

- brain metastases

- immunotherapy

- neurosurgery $•$ radiation

therapy • systemic therapy

- targeted therapy
Melanoma is the third most common solid tumor to metastasize to the brain, with a prevalence of $20-40 \%$ in clinical series and up to $75 \%$ in autopsy series $[1,2]$. The development of brain metastases (BMs) is a devastating event in the course of advanced melanoma. Historically, patients with advanced melanoma and active BMs have dismal prognosis. Thus, improving survival outcome while preserving quality of life for this patient subset has been the most important goal of multidisciplinary research efforts.

The management of melanoma BMs continues to rely heavily on surgical and radiotherapeutic interventions, which have become safer and more effective with modern technology. Until recently, systemic treatments for melanoma patients with BMs are primarily used as palliative therapy, with options limited to a few cytotoxic agents that can penetrate the blood-brain barrier (BBB). Recent insights into the molecular basis of melanoma and the intricate regulatory mechanisms of tumor immunity have expanded the pharmacologic options for this subset of patients. This review aims to provide an update on recent progress in the treatment of patients with advanced melanoma and BMs.

\section{Risk factors \& prognosis}

The prognosis of advanced melanoma involving the CNS is grim, with a median overall survival (OS) of 3.8-5.2 months [2-4]. In the effort to develop more effective therapies for melanoma BMs, several studies were carried out to identify the risk factors for BM development as well as the prognostic factors for patients with melanoma BMs. Various clinicopathologic features have been linked to BM development. Zakrzewski et al. showed the presence of ulceration in the primary lesion was predictive of BM development [5]. Bedikian et al. identified M stage (M1b or M1c vs M1a + III) and elevated lactic acid dehydrogenase levels as the predictive factors for BMs at diagnosis of stage III or IV melanoma [6]. Qian et al. associated tumor thickness, presence of ulceration and advanced stage (III or IV vs I or II) with high risk of BM development [7]. In the era of genomic medicine, evidence associating molecular abnormalities with CNS metastases is emerging. Jakob et al. linked NRAS or BRAF mutations with increased risk of CNS involvement [8]. In addition, loss of PTEN expression has recently been associated with a shorter time to BM development in patients with $B R A F \mathrm{~V} 600$-mutant stage IIIB or
IIIC melanoma [9]. These risk factors, however, will require rigorous validation in larger patient cohorts before they can be used as predictors of $\mathrm{BM}$ and alter current surveillance and preventative strategies [10].

Another aim of the aforementioned retrospective studies was to identify prognostic factors influencing OS from the time of BM occurrence. Multiple BMs, leptomeningeal involvement, coexistence of extracranial disease, elevated lactic acid dehydrogenase, poor performance status, and head and neck primary lesions were among the identified indicators of unfavorable OS after BM development $[2,4-5,11]$. Furthermore, several therapeutic interventions, in other words, surgery, stereotactic radiosurgery (SRS), temozolomide-based chemotherapy and immunotherapy, have been correlated with improved survival in patients with BMs [3-4,11-13]. Nevertheless, these results are from retrospective analyses, which are not able to control for potential confounders and selection biases. In addition, the patient- and/or disease-specific characteristics that steer treating physicians to select different treatment strategies in these studies make it difficult to discern the survival benefit of each therapeutic modality [10].

To differentiate the relative influence of pretreatment patients' characteristics from treatment effects on the OS of patients with BMs, the Radiation Therapy Oncology Group (RTOG) developed a prognostic index named recursive partitioning analysis (RPA) based on a retrospective analysis of 1200 patients treated in three RTOG BM trials [14]. Of note, these studies enrolled participants regardless of primary cancer diagnosis, with majority of patients having breast or lung cancers. The validated RTOG RPA groups patients into three prognostic categories: class 1, including patients younger than 65 years of age with Karnofsky performance status (KPS) $\geq 70$, controlled primary and no extracranial disease, has the best OS (median OS: 7.1 months); class 3, comprising patients with KPS $<70$, has the worst outcome (median OS: 2.3 months) and class 2 , with the remaining patients, has intermediate prognosis (median OS: 4.2 months) $[14,15]$.

To minimize the subjectivity in appraising the status of primary tumor control and to include the number of BM lesions in prognostic scoring system, the RTOG subsequently developed the Graded Prognostic Assessment (GPA), which assigned patients into four prognostic classes based on age, KPS, number of brain lesions and 
presence or absence of extracranial disease [16]. The GPA has also been validated and, more importantly, tailored to diagnosis-specific prognostic variables for five different cancers, including breast, lung, gastrointestinal, melanoma and renal cell carcinoma [17]. The melanoma-specific GPA score, calculated based on KPS and the number of BMs, helps clinicians individualize treatment plans or to stratify participants of clinical trials evaluating BM therapies.

\section{Treatment}

- Local therapy

Selection of treatment modalities for melanoma BMs relies upon a comprehensive multidisciplinary evaluation of patient's performance status, the number, size and location of $B M s$, the extent of extracranial disease and $B R A F$ mutation status. At present, aggressive local therapy to completely eradicate all brain lesions with surgical resection and/or SRS, with or without whole brain radiation therapy (WBRT), is the standard of care in patients with favorable prognosis, based on data from many retrospective and prospective studies suggesting an improved survival outcome compared with best supportive care or WBRT alone [4,11,18-19].

\section{Surgery}

Surgical resection of metastatic brain disease is indicated for histologic diagnosis, if not yet ascertained, or rapid decompression of intracranial mass effect. In addition, surgical resection of solitary metastatic brain lesion has been shown to improve OS in two randomized trials $[18,19]$. Patchell et al. compared the efficacy of surgical resection followed by WBRT with WBRT alone in 48 patients with single BM from a number of tumor types, among whom three had advanced melanoma [18]. The median OS were 40 weeks in the surgery arm and 15 weeks in radiotherapyalone arm $(\mathrm{p}<0.01)$. Local disease control and functional independence were also improved with surgery followed by WBRT. The complication rates were similar between the two groups. Thirty-day mortality rate was $4 \%$ in each arm. The 30-day morbidity rates were 8 and $17 \%$ in the surgery-WBRT and WBRT-alone groups, respectively.

Another randomized study by Vecht $e t$ al. confirmed prolonged OS with surgery followed by WBRT compared with WBRT alone in 63 patients with single BM, among whom six had advanced melanoma (median OS: 10 months vs 6 months; $p=0.04)$ [19]. Functionally independent survival was also longer in the surgeryplus WBRT arm. The 1-month mortality rates were 9 versus $0 \%$ in the combined treatment and WBRT-alone arms, respectively. The early deaths in the combined treatment group occurred in patients with infratentorial lesions. Morbidities were comparable between the two arms.

However, Mintz et al. reported no OS difference in 84 patients with single BM in a randomized study of WBRT with or without surgical resection [14]. Of note, a higher percentage of patients with poor prognosis, low KPS and/or coexisting extracranial disease, were enrolled in this study as compared with the previous two trials. This factor may account for the negative results of the study [20].

For patients with multiple metastatic brain lesions, the OS benefit of complete surgical removal of BMs is supported only by retrospective studies [21,22]. Bindal et al. compared survival outcome of 30 patients with multiple $\mathrm{BMs}$ with some BM lesions resected (group A) versus 26 patients with all $\mathrm{BM}$ lesions resected (group B) [21]. Results were also compared with the historical data of 26 patients with resected single BM lesion (group C), who best matched those in group B. Median OS were 6, 14 and 14 months in group $A, B$ and $C$, respectively ( $A$ vs $B, p=0.03$; $A$ vs $C, p=0.012$; $B$ vs $C, p>0.5)$. Konstadoulakis et al. reported higher 1-year survival rate with surgical resection, compared with radiotherapy, chemotherapy or supportive care, in patients with single or multiple BMs [22]. These data suggest multiple craniotomies can be safely performed, and complete removal of all BM lesions may improve survival.

Most of the aforementioned studies are not melanoma specific, and data for the survival benefit of neurosurgery in patients with melanoma BMs are limited. These retrospective series suggest that surgical resection of BMs potentially prolongs OS compared with WBRT or supportive care in select patients, with good performance status, limited or no extracranial disease, and BMs amenable to safe and complete resection $[4,11,22]$. Please refer to Table 1 for summary of key neurosurgery studies.

\section{Radiation therapy}

Melanoma is considered resistant to low doses of radiation delivered in standard fractionated courses of radiotherapy $[23,24]$. Consequently, the 


\begin{tabular}{|c|c|c|c|c|c|c|c|}
\hline Study & No. of BMs & $\begin{array}{l}\text { No. of patients } \\
\text { (\% melanoma) }\end{array}$ & Treatment (n) & $\begin{array}{l}\text { Local } \\
\text { control (\%) }\end{array}$ & $\begin{array}{l}\text { Median } \\
\text { functionally } \\
\text { independence } \\
\text { survival }\end{array}$ & $\begin{array}{l}\text { Median overall } \\
\text { survival }\end{array}$ & Ref. \\
\hline Patchell et al. & Solitary & $48(6)$ & $\begin{array}{l}\text { Surgery + WBRT (25) } \\
\text { WBRT }\end{array}$ & $\begin{array}{l}20^{+} \\
52^{+}\end{array}$ & $\begin{array}{l}38 \text { weeks }^{\dagger} \\
8 \text { weeks }^{\dagger}\end{array}$ & $\begin{array}{l}40 \text { weeks }^{\dagger} \\
15 \text { weeks }^{\dagger}\end{array}$ & {$[18]^{\ddagger}$} \\
\hline Vecht et al. & Solitary & $63(10)$ & $\begin{array}{l}\text { Surgery + WBRT (32) } \\
\text { WBRT }\end{array}$ & NR & $\begin{array}{l}7.5 \text { months }^{\S} \\
3.5 \text { months }^{\S}\end{array}$ & $\begin{array}{l}10 \text { months }^{\dagger} \\
6 \text { months }^{\dagger}\end{array}$ & {$[19]^{\ddagger}$} \\
\hline Mintz et al. & Solitary & $84(5)$ & $\begin{array}{l}\text { Surgery + WBRT (41) } \\
\text { WBRT }\end{array}$ & NR & No difference & $\begin{array}{l}6.28 \text { months } \\
5.62 \text { months }\end{array}$ & {$[20]^{\dagger}$} \\
\hline Bindal et al. & Multiple & $56(45)$ & $\begin{array}{l}\text { Resection of some BMs (30) } \\
\text { Resection of all BMs (26) } \\
\text { Matched control (solitary BM) (26) }\end{array}$ & $\begin{array}{l}\text { NR } \\
4 " \\
8 "\end{array}$ & NR & $\begin{array}{l}6 \text { months }^{\dagger} \\
14 \text { months }^{\dagger} \\
14 \text { months }\end{array}$ & {$[21]^{*}$} \\
\hline $\begin{array}{l}\text { Konstadoulakis } \\
\text { et al. }\end{array}$ & Mixed & $136(100)$ & $\begin{array}{l}\text { Surgery (32) } \\
\text { WBRT or chemotherapy or both (75) } \\
\text { No therapy (29) }\end{array}$ & NR & NR & $\begin{array}{l}6.67 \%^{+, t+} \\
3.45 \% \%^{t, t+}\end{array}$ & {$[22]$} \\
\hline \multicolumn{8}{|c|}{$\begin{array}{l}\text { †Statistically significant difference. } \\
\text { "Randomized. } \\
{ }^{5} \mathrm{p}=0.06 \text {. } \\
\text { 'Percentage local relapse. } \\
\text { "Retrospective. } \\
{ }^{+} \text {At } 1 \text { year. } \\
\text { BM: Brain metastases; NR: Not repo }\end{array}$} \\
\hline
\end{tabular}

use of radiation in the treatment of melanoma, particularly when metastatic to the relatively radiation-sensitive normal tissues of the CNS, has often been reserved for palliation of unresectable disease or in the adjuvant/postoperative setting. With the advent of improved target localization and radiation delivery techniques, radiation therapy (RT) is often the first choice for local control of brain disease in patients with metastatic melanoma.

\section{Whole brain radiation therapy}

WBRT delivers small daily doses of RT, typically from 2 to 3 Gy per fraction, for ten to 20 fractions and a total dose of 30-40 Gy, to the entire intracranial contents, thereby treating the known, visible BMs as well as any microscopic disease elsewhere in the brain not detected on routine imaging. In one retrospective analysis of 41 patients with radiation resistant BMs, of which 23 had melanoma, Brown et al. showed that 6-month actuarial local control (LC) in the brain was improved to $100 \%$ with WBRT from $85 \%$ without WBRT, and that elsewhere failure in the brain was reduced from $64 \%$ without WBRT to $17 \%$ with WBRT [24]. This finding of significantly improved intracranial disease control with WBRT was also demonstrated in two large Phase III studies [25,26]. Progression of brain disease was identified as the cause of death in $44 \%$ of patients not receiving WBRT and in $28 \%$ of those treated with WBRT in the European Organization for Research and Treatment of Cancer trial [26]. Of note, however, only $5 \%$ of subjects were noted to have melanoma in this study [26], and the percentage of patients having melanoma was not reported in the other Phase III study comparing WBRT with SRS [25]. Results of an international Phase III trial of WBRT following local therapy of intracranial metastases from melanoma being conducted by the Australia and New Zealand Melanoma Trials Group and the Trans Tasman Radiation Oncology Group are still pending [27].

In an attempt to overcome the relative resistance of melanoma to standard fractionated radiation used in WBRT, various approaches to increase the radiation sensitivity of melanoma BMs have been investigated. One such approach has examined the benefit of RT dose escalation. In one retrospective study of 87 melanoma patients with multiple BMs, Hauswald et al. reported a near doubling of median OS from 3.1 months after standard $30 \mathrm{~Gy}$ in 10 fractions to 5.6 months following dose escalated WBRT of $40 \mathrm{~Gy}$ in 20 fractions ( $\mathrm{p}=0.003$ ) [28]. The results from this analysis are confounded by the use of other therapies to improve LC including craniotomy or RT boost. Improved OS following higher dose WBRT was shown in another retrospective analysis of 51 patients with BMs from melanoma [29]. In this study, patients receiving 
40 Gy in 20 fractions or 45 Gy in 15 fractions had $20 \%$ 1-year OS, compared with only $4 \%$ of those receiving $30 \mathrm{~Gy}$ in 10 fractions $(\mathrm{p}=0.009)$. Other important characteristics associated with improved OS in this group of patients included the presence of $<4 \mathrm{BMs}$, no extracranial disease and RPA class 1 . Table 2 summarizes the key findings of studies evaluating the utility of WBRT in the management of melanoma BMs.

Another approach that has been evaluated to improve the therapeutic effect of WBRT in the management of intracranial metastases from melanoma is the concurrent use of systemic agents known to penetrate the BBB during RT to act as potential radiosensitizers. Specific agents including temozolomide [32-34], fotemustine [35] and ipilimumab [36], have been investigated with WBRT with mixed results. These analyses are addressed in more detail in the chemotherapy section below.

In addition to the work by Rades et al. [29], several other investigators have demonstrated longer OS following WBRT in melanoma patients whose extracranial disease is controlled [30-31,37], supporting the hypothesis that extent of disease outside the brain is an important measure of life-limiting disease burden in these patients. Consequently, presence or absence of extracranial disease has been included in newer scoring systems for radiation-resistant brain metastases to identify subjects who may benefit from WBRT with dose escalation and/or stereotactic radiosurgical boosting, discussed below [37,38].
Stereotactic radiosurgery

SRS is a technique initially developed by the Swedish neurosurgeon Lars Leksell to deliver high doses of radiation to well-defined, small intracranial targets in a single fraction using a minimally invasive head frame externally affixed to the patient's cranium. Proposed advantages of SRS over WBRT in the management of BMs from melanoma include improved LC of radiation-resistant tumors by the ablative RT dose delivered by SRS, decreased toxicity associated with the focal brain SRS treatment and minimal interruption of systemic therapy regimens by the 1-day SRS therapy.

Several prospective investigations have shown improved LC of BMs when SRS is added to WBRT. An early study of patients with two to four BMs by Kondziolka et al. was stopped early due to significant reduction in 1-year local failure from $100 \%$ following 30 Gy in 12 fractions WBRT alone to $8 \%$ with addition of SRS boost $(p=0.0016)$ [39]. Melanoma was the second most common histology after lung carcinoma, and there was no association identified between tumor type and LC in this study $(\mathrm{p}=0.85)$. In a larger, Phase III trial by the RTOG, RTOG 9508, patients with one to three BMs receiving SRS after 37.5 Gy in 15 fractions WBRT had $82 \%$ 1-year LC compared with $71 \%$ in WBRT alone $(p=0.01)[40]$. Lung cancer again comprised the majority of subjects in this trial, and only $4 \%$ had melanoma. The improved intracranial disease control with SRS after WBRT

\begin{tabular}{|c|c|c|c|c|c|}
\hline Study & $\begin{array}{l}\text { No. of } \\
\text { patients (\% } \\
\text { melanoma) }\end{array}$ & Treatment (no.) & Local control (\%) & $\begin{array}{l}\text { Median overall } \\
\text { survival (months) }\end{array}$ & Ref \\
\hline $\begin{array}{l}\text { Brown } \\
\text { etal. }\end{array}$ & $41(56)$ & $\begin{array}{l}\text { SRS alone } 18 \mathrm{~Gy}(15) \\
\text { SRS + WBRT 30-50Gy/10-25 fx }\end{array}$ & $\begin{array}{l}\text { SRS alone: } 85^{\dagger} \\
\text { SRS + WBRT: } 100^{\text {t‡ }}\end{array}$ & 14.2 & [24] \\
\hline $\begin{array}{l}\text { Hauswald } \\
\text { etal. }\end{array}$ & $87(100)$ & $\begin{array}{l}\text { WBRT } \\
30 \mathrm{~Gy} / 10 \mathrm{fx}(56) \\
40 \mathrm{~Gy} / 20 \mathrm{fx}(31)\end{array}$ & NR & $\begin{array}{l}\text { All: } 3.5 \\
30 \text { Gy: } 3.1 \\
40 \text { Gy: } 5.6^{\ddagger}\end{array}$ & {$[28]$} \\
\hline $\begin{array}{l}\text { Rades } \\
\text { et al. }\end{array}$ & $51(100)$ & $\begin{array}{l}\text { WBRT } \\
30 \mathrm{~Gy} / 10 \mathrm{fx}(33) \\
40 \mathrm{~Gy} / 20 \mathrm{fx}(11) \\
45 \mathrm{~Gy} / 15 \mathrm{fx}(7)\end{array}$ & $\begin{array}{l}30 \mathrm{~Gy}: 23^{\dagger} \\
40-45 \mathrm{~Gy}: 50^{\dagger, \neq}\end{array}$ & $\begin{array}{l}30 \text { Gy: } 27 \%^{\dagger} \\
40-45 \text { Gy: } 50 \%^{+\ddagger}\end{array}$ & [29] \\
\hline $\begin{array}{l}\text { Ellerhorst } \\
\text { et al. }\end{array}$ & $87(100)$ & $\begin{array}{l}\text { WBRT } \\
15-50 \text { Gy/5-25 fx }\end{array}$ & NR & 4.75 & {$[30]$} \\
\hline Dyer et al. & $147(100)$ & $\begin{array}{l}\text { SRS } \\
+ \text { Up-front WBRT (39) } \\
+ \text { Salvage WBRT (33) }\end{array}$ & NR & 7.3 & [31] \\
\hline
\end{tabular}


in both studies did not translate into significant OS improvement. However, the extent of extracranial disease and RPA class were prognostic of survival outcomes in these patients. Although patients with single BM were excluded from the earlier trial, the subset of patients with solitary BM treated with WBRT and SRS in the RTOG trial had a median survival of 6.5 months, compared with 4.9 months in the WBRT-alone group ( $\mathrm{p}=0.0393)$.

SRS as a single treatment modality has been shown by multiple studies to improve LC of radioresistant BMs compared with WBRT alone [41-45]. Mori and collaborators examined outcomes of 60 melanoma patients with BMs, nine of which were treated with SRS alone [42]. The rate of LC of 72 evaluable tumors was $90 \%$ in this analysis. In another retrospective analysis of 35 melanoma patients with a total of 70 BMs treated with SRS alone, Grob et al. demonstrated comparable LC rates of evaluable lesions through 12 months of follow-up as was reported by Mori et al. [41]. Selek et al. performed a similar analysis of 103 melanoma patients with $153 \mathrm{BMs}$ treated at MD Anderson Cancer Center [44]. Of these patients, 61 received SRS alone. In this investigation, LC following SRS was dependent on lesion size, with $75 \%$ LC rate in tumors measuring $2 \mathrm{cc}$ or less versus $42 \%$ in larger lesions. Survival was greatest for patients with single lesion in both Mori and Grob's analyses, but number of metastases was not found to be prognostic for survival in the MD Anderson study. Despite these findings of improved LC of melanoma BMs with SRS, other studies that include similarly treated patients with various radiation-resistant histology primary tumors demonstrated that patients with melanoma had a higher incidence of neurologic death $[29,45]$.

In the largest retrospective analyses of SRS for melanoma BMs, Mathieu et al. reported on outcomes of 244 patients with 754 brain lesions treated with radiosurgery [46]. As was observed in the other studies, LC of BMs following SRS was high, exceeding $86 \%$ for all lesions. Both tumor volume and prior hemorrhage were associated with worse LC, on multivariate analysis. The median OS in this series was 5.3 months, and survival was found to be significantly improved in those patients with controlled extracranial disease, KPS $\geq 90 \%$ and solitary BMs. Approximately half $(n=110)$ of the subjects in this study received WBRT prior to SRS. The median survival in this subgroup was only 4.7 months, compared with 5.7 months for patients with no prior WBRT ( $\mathrm{p}=0.01$ on log-rank test). While this finding might be interpreted as patients receiving the combined WBRT and SRS have worse outcome, it more likely reflects, in part, the delayed delivery of SRS in these patients, since median survival of patients receiving WBRT and SRS was 8.6 months versus 7.2 months with SRS alone, when calculated from the date that BMs were initially detected. Table 3 summarizes the key findings of studies investigating the utility of SRS in the management of melanoms BMs.

Compared with WBRT, SRS has been found to have less treatment-associated toxicity and morbidity, most notably with respect to preservation of neurocognitive function. A landmark Phase III trial designed to evaluate neurocognitive function at 4 months following treatment of patients with 1-3 BMs with WBRT plus SRS or SRS alone by Chang et al. was closed early due to a doubling of decline in memory and learning functions being identified in the group receiving WBRT as part of their therapy compared with the SRS only group (52 vs 24\%) [47]. OS was higher in the patients receiving SRS alone, despite improved LC in those receiving the combined WBRT and SRS treatment, recognizing that the study was not powered for these secondary end points. Similar to the RTOG 9508 study, the percentage of patients with melanoma in the study by Chang et al. was only 3-4\%. A more recent prospective pilot study comparing neurocognitive outcomes of 20 patients receiving WBRT versus seven receiving SRS alone conducted by Onodera and colleagues revealed both immediate and delayed memory to be significantly reduced at 4 months in the former group [48]. Memory function in the WBRT-treated patients improved to baseline at 8 months, but declined again in those subjects followed for 12 months. In the SRS-treated subjects, there was no change in the measured neurocognitive functions over this period of time. None of these patients were specifically identified as having melanoma, although 11\% were categorized as having tumor histology other than lung or breast cancer.

Randomized controlled clinical trials investigating SRS alone versus WBRT have limited the number of BMs per patient to four or less [25,47], since the number of BMs has commonly been considered a surrogate for disease failure elsewhere in the brain. Recent analyses, however, 


\begin{tabular}{|c|c|c|c|c|c|c|}
\hline Mori et al. & $60(100)$ & $\begin{array}{l}\text { GK salvage (12) } \\
\text { WBRT + GK (36) } \\
\text { GK alone (12) }\end{array}$ & $16.4(10-20)$ & 90 & 7.0 & [42] \\
\hline Herfarth et al. & $64(100)$ & $\begin{array}{l}\text { Linac } 18(10-24) \\
\text { Linac salvage (30) }\end{array}$ & $20(15-22)$ & $81^{\ddagger}$ & 10.6 & {$[43]$} \\
\hline Selek et al. & $103(100)$ & $\begin{array}{l}\text { WBRT + Linac (12) } \\
\text { Linac alone (61) }\end{array}$ & $18(10-24)$ & $49^{\ddagger}$ & $25.2 \% \neq$ & [44] \\
\hline Chang et al. & $189(54)$ & $\begin{array}{l}\text { Linac } \\
\text { WBRT + GK (110) }\end{array}$ & $18(10-24)$ & $47^{\ddagger}$ & 6.7 & [45] \\
\hline \multicolumn{7}{|l|}{$\begin{array}{l}{ }^{\star} \text { At } 3 \text { months; } \\
{ }^{*} \text { At } 1 \text { year. } \\
\text { BM: Brain metas }\end{array}$} \\
\hline
\end{tabular}

support the concept that the total volume of intracranial disease is more predictive of outcome than the total number of lesions [44-45,49-51]. A recent case-matched analysis of 360 patients treated with SRS alone for 2-9 BMs versus the same number of similarly treated patients with at least ten BMs by Yamamoto et al. revealed comparable median and neurologic death-free survival in both groups [52]. Importantly, there were also no significant differences in neurologic deterioration or treatment-related complications between these groups. The proportion of patients in the Yamamoto study with melanoma was not reported, although $6 \%$ of subjects had primary tumor sites other than lung, breast, alimentary canal or kidney. General acceptance of these findings will likely lead to a paradigm shift in the treatment of melanoma patients with multiple BMs away from traditional WBRT and toward ablative SRS. In support of this approach, addition of WBRT after SRS has not been shown to improve OS in several studies [13,24,31,53].

\section{- Systemic therapy}

Prior to 2011, dacarbazine (DTIC) and IL-2 were the only two systemic therapeutic agents approved by the US FDA for the treatment of advanced melanoma. Unfortunately, these pharmacologic options had minimal utility in the management of patients with BMs. Dacarbazine achieves negligible cerebrospinal fluid concentration [54]; thus, it is ineffective against CNS metastases. High-dose IL-2 is typically avoided in patients with active BMs due to concern over worsening cerebral edema [55]. Until recently, the mainstay of systemic therapy for melanoma patients with BMs has been temozolomide or fotemustine.

\section{Chemotherapy}

Temozolomide

Temozolomide is a congener of DTIC with $100 \%$ oral bioavailability and similar clinical activity [56]. Unlike DTIC, temozolomide achieves clinically sufficient cerebrospinal fluid concentration; therefore, it is commonly used as an alternative to DTIC in patients with active BMs [57].

A large multicenter Phase II trial evaluated temozolomide $150-200 \mathrm{mg} / \mathrm{m}^{2}$ daily for 5 days every 28 days in 151 patients with metastatic melanoma with active BMs [58]. Temozolomide demonstrated an intracranial tumor response and disease stabilization rates of 7 and 29\%, respectively. The survival impact of temozolomide was marginal, with a median progressionfree survival (PFS) of approximately 1 month and median OS of 3.2 months [58]. A protracted dosing regimen of temozolomide, $75 \mathrm{mg} / \mathrm{m}^{2}$ daily for 6 weeks followed by 2 weeks of rest, in combination with thalidomide, a compound with antiangiogenic and immunomodulatory activities, was also explored in melanoma patients with CNS involvement [59]. Despite the initial encouraging results, a confirmatory multicenter, Phase II study conducted by the Cancer 
and Leukemia Group B to evaluate the combined extended-dose temozolomide and thalidomide in melanoma patients with BMs was terminated prematurely for concerns over safety and efficacy [60]. No objective response was noted in the initial 16 patients, but life-threatening thromboembolic events were observed in 31\% of patients.

Based on the positive result of concurrent temozolomide and WBRT in patients with primary brain tumors, Margolin et al. evaluated the use of temozolomide with WBRT in patients with melanoma BMs [34]. A total of 31 patients were treated with WBRT at 30 Gy in ten fractions, and protracted-dose temozolomide at $75 \mathrm{mg} / \mathrm{m}^{2}$ daily for 6 weeks followed by 4 weeks off. Three patients achieved a CNS objective response (9.7\%; 95\% CI: $2.0-25 \%)$. The median PFS and OS were 2 and 6 months, respectively. Concurrent temozolomide and WBRT offered negligible advantage in terms of intracranial antitumor activity in patients with melanoma BMs.

\section{Fotemustine}

Fotemustine is a nitrosourea licensed in Europe for the treatment of disseminated malignant melanoma. Able to diffuse across the BBB, fotemustine has been extensively evaluated in patients with advanced melanoma and BMs, with intracranial responses rates of $25-28 \%$ in Phase II trials [61-63].

However, CNS activity of this agent was low when compared with dacarbazine in a Phase III study of 229 patients with disseminated melanoma, with or without BMs [64]. Objective responses in the brain were 5.9 and $0 \%$ in the fotemustine- and dacarbazine-treated groups, respectively. Combining fotemustine with WBRT did little to improve its efficacy. In a randomized Phase III trial evaluating fotemustine plus WBRT versus fotemustine monotherapy in 69 patients with stage IV melanoma and BMs, there were no statistically significant differences in intracranial responses (10.0 vs $7.4 \%$ ), intracranial disease control (47 vs 30\%) or OS (105 vs 86 days) between the two treatment arms [35].

Thus, although considered the most promising systemic treatment options for melanoma BMs, temozolomide and fotemustine, with or without WBRT, had minimal impact on the prognosis in patients with advanced melanoma involving the CNS. Please refer to Table 4 for summary of key chemotherapy studies.
Immunotherapy

Recent understanding of the molecular basis of melanoma and the intricate regulatory mechanisms of tumor immunity has added six novel agents, ipilimumab, vemurafenib, dabrafenib, trametinib, pembrolizumab and nivolumab, for the treatment of advanced melanoma. With new evidence refuting the absolute impenetrable and immuno-privileged nature of the CNS environment at the sites of metastatic lesions, these therapeutic advances have revolutionized the systemic treatment strategy for melanoma BMs [70-72].

\section{Ipilimumab}

Cytotoxic T-lymphocyte antigen 4 (CTLA-4) is an inhibitory immune checkpoint receptor that plays a critical role in regulating the adaptive immune response by interrupting the costimulatory signal essential for T-cell activation. Ipilimumab is a fully human IgG1 monoclonal antibody that blocks CTLA-4. By negating the inhibitory action of CTLA-4, ipilimumab augments $\mathrm{T}$-cell responses to tumor antigens, resulting in immune-mediated antitumor activity. Since its approval by regulatory agencies worldwide, ipilimumab, administered at $3 \mathrm{mg} / \mathrm{kg}$ every 3 weeks for a total of four doses, has become a standard treatment option for patients with extracranial advanced melanoma.

Intracranial antitumor activity of ipilimumab, at $10 \mathrm{mg} / \mathrm{kg}$ for four doses followed by a maintenance phase, has been demonstrated in a multicenter Phase II study by Margolin et al. (Table 4) [65]. Patients were enrolled in two cohorts: neurologically asymptomatic patients who did not require corticosteroids were assigned to cohort A, whereas those with neurological symptoms and on a stable dose of corticosteroids went to cohort B. The antitumor activity of ipilimumab was similar in the brain and at extracranial sites. Out of 51 patients in cohort A, eight achieved partial response (PR) and four had stable disease (SD) in the brain. The median OS for cohort A was 7.0 months (95\% CI: 4.1-10.8 months), with a 2-year OS rate of $26 \%$ (95\% CI: $14-39 \%$ ). The results were less favorable in cohort B $(n=21)$, with one complete response and one SD in the brain. The median OS for cohort B was 3.7 months (95\% CI: $1.6-7.3$ months), with an OS rate of 10\% (95\% CI: $0-22 \%)$ at 2 years. From a safety standpoint, the immune-related adverse events associated with ipilimumab were similar to prior 
Table 4. Summary of key systemic therapy studies.

\begin{tabular}{|c|c|c|c|c|c|c|}
\hline Study & No. of patients & Treatment & Intracranial response (\%) & $\begin{array}{l}\text { Median progression- } \\
\text { free survival (months) }\end{array}$ & $\begin{array}{l}\text { Median overall } \\
\text { survival (months) }\end{array}$ & Ref. \\
\hline \multirow[t]{2}{*}{ Agarwala et al. } & \multirow[t]{2}{*}{151} & \multirow[t]{2}{*}{ Temozolomide } & Chemo-naive: 7 & 1.2 & 3.5 & \multirow[t]{2}{*}{ [58] } \\
\hline & & & Prior chemo: 1 & 1.0 & 2.2 & \\
\hline Jacquillat et al. & 39 & Fotemustine & 28.2 & NR & 26 weeks & {$[61]$} \\
\hline \multirow[t]{2}{*}{ Avril et al. } & \multirow[t]{2}{*}{229 (43 with BMs) } & Fotemustine & 5.9 & \multirow[t]{2}{*}{ NR } & \multirow[t]{2}{*}{ NR } & \multirow[t]{2}{*}[64]{$^{\dagger}$} \\
\hline & & Dacarbazine & 0 & & & \\
\hline Queirolo et al. & 146 & Ipilimumab & NR & 2.8 & 4.3 & {$[66]^{\S}$} \\
\hline Dummer et al. & 24 & Vemurafenib & 16 & 3.9 & 5.3 & {$[67]$} \\
\hline \multirow[t]{2}{*}{ Kefford et al. } & \multirow[t]{2}{*}{146} & \multirow[t]{2}{*}{ Vemurafenib } & No prior local therapy: 21 & 3.74 & 7.06 & \multirow[t]{2}{*}[68]{} \\
\hline & & & Prior local therapy: NR & 3.94 & 9.53 & \\
\hline Long et al. & 172 & Dabrafenib & No prior local therapy: $39.2^{n}$ & 16.1 weeks" & 33.1 weeks" & {$[69$} \\
\hline
\end{tabular}

experience. The most common CNS-related toxicities were grade 1 or 2 headache, dizziness and confusion. Three cases of brain edema and one case of intracranial hemorrhage were reported and considered disease related.

This encouraging result has led to the NIBIT-M1 trial, in which ipilimumab, at $10 \mathrm{mg} / \mathrm{kg}$ for four doses with a maintenance phase, was combined with fotemustine to treat 20 patients with asymptomatic BMs [73]. The majority of those patients had 2-3 intracranial lesions; a third of them had received prior local therapy including SRS or WBRT. At followup scans, BMs were reduced or stable in six patients and no longer visible in five others. The median PFS and OS were 4.5 (95\% CI: 3.4-7.1) and 13.4 months (95\% CI: $10.0-$ not evaluable), respectively. Approximately half of the study population remained alive at 1 year. The most common toxicities of the combination were immune-related adverse events and myelosuppression. Five patients experienced CNSrelated events, that is, headache, seizure and hemorrhage, which were attributed to disease progression in the brain.

The currently approved dosing schedule of ipilimumab, $3 \mathrm{mg} / \mathrm{kg}$ for four doses, also demonstrates intracranial activity, as shown in 146 patients with asymptomatic BMs identified from the Italian cohort of the ipilimumab expanded access program (Table 4) [66]. The immune-related response criteria were used to evaluate global disease response. Among 26 patients who were on corticosteroid at baseline, two achieved PR and four had SD, for a global disease control rate of $15 \%$. In patients not taking steroid at baseline, the global response rate and disease control rate were 12 and 29\%, respectively. With a median follow-up of 20 months, the median PFS and OS were 3.1 and 4.3 months, respectively, for the entire study population. The OS rate at 1 year was $20 \%$. In multivariate analysis, steroid use, age $<60$ years and poor Eastern Cooperative Oncology Group performance status were significantly associated with decreased survival. Ten (6.9\%) patients experienced CNS adverse events, such as headache, seizure and hemorrhage, of which $1 \%$ may be ipilimumab related.

Thus, ipilimumab appears safe and effective in patients with advanced melanoma and BMs who do not require corticosteroids for CNSrelated symptoms. Although the fraction of patients benefiting from ipilimumab is small, the ability of this agent to exert durable intracranial disease control is intriguing. Considerable research efforts are ongoing to develop ipilimumab in combination with other modalities, such as radiotherapy (NCT01703507) [74], chemotherapy (NCT02460068) [75], BRAF inhibitors or other immunomodulatory agents (NCT02320058) [76], in the management of melanoma patients with BMs.

\section{Anti-PD-1 monoclonal antibodies}

$\mathrm{PD}-1$ receptor, expressed on activated $\mathrm{T}$ and $\mathrm{B}$ cells, NK cells and monocytes, is another 
important negative regulator of the immune system. Engagement of PD-1 to its primary ligand, PD-1 ligand 1 (PD-L1 or B7-H1), inhibits T-cell effector function, ultimately inducing T-cell exhaustion and deletion [66,77]. Upregulation of PD-L1 expression has been demonstrated in the tumor microenvironment of many human cancers, including melanoma. Monoclonal antibodies targeting the PD-1: PD-L1 pathway are actively investigated as immunotherapeutic strategies for various malignancies [77,78]. Nivolumab (BMS 936558, MDX-1106) and pembrolizumab (MK-3475), the two anti-PD-1 monoclonal antibodies, have demonstrated robust and durable clinical response in patients with advanced melanoma $[79,80]$. In 2014, both agents were licensed in the USA as second- or third-line therapy for patients with advanced disease refractory to ipilimumab and BRAF inhibitors.

The intracranial activity of nivolumab was suggested by a case report by Lipson and colleagues [81]. A 76-year old patient with disseminated renal cell carcinoma refractory to multiple lines of therapy was enrolled in a first-in-human, Phase I dose-escalation study of nivolumab. His disease achieved a PR after three doses of nivolumab $10 \mathrm{mg} / \mathrm{kg}$. Restaging scans obtained 6 months later revealed a new $1.4-\mathrm{cm}$ hemorrhagic brain lesion with peritumoral edema in the right frontal lobe consistent with intracranial metastasis. Pathologic evaluation of the resected lesion demonstrated a lymphocytic infiltrate and no evidence of viable tumor, suggesting of complete pathological response. Studies to assess the safety and efficacy of nivolumab or pembrolizumab in patients with metastatic melanoma and untreated BMs are being planned (NCT02085070 and NCT02320058) [76,82].

\section{Dual checkpoint blockade}

Since CTLA-4 and PD-1 are nonoverlapping regulators of the activation and effector phases of T-cell immunity, concurrent administration of ipilimumab and nivolumab has been explored in patients with advanced melanoma in a Phase I study, demonstrating remarkable clinical activity [83]. A multicenter Phase II trial to evaluate this combination in patients with untreated melanoma BMs is being planned (NCT02320058) [76].

\section{Targeted therapy}

BRAF and MEK are components of the MAPK pathway, which regulate cell growth, differentiation and survival. Approximately $50 \%$ of melanomas carry activating mutations in the $B R A F$ gene, among which $B R A F^{\mathrm{V} 600 \mathrm{E}}$ and $B R A F^{\mathrm{V} 600 \mathrm{~K}}$ are the two most prevalent mutant genotypes $[84,85] . \mathrm{BRAF}^{\mathrm{V} 600 \mathrm{E}}$ and $\mathrm{BRAF}^{\mathrm{V} 600 \mathrm{~K}}$ kinases are constitutively active, leading to continual MAPK signaling and uncontrolled tumor growth.

Vemurafenib and dabrafenib are small molecule kinase inhibitors of mutant $\mathrm{BRAF}^{\mathrm{V} 600}$; while trametinib is an inhibitor of MEK, the protein immediately downstream of BRAF in the MAPK pathway. By blocking the mutationdriven constitutive MAPK pathway activation, these agents have significantly improved antitumor response and survival for patients with $B R A F^{V^{600}}$-mutant advanced melanoma in randomized Phase III studies, leading to the approval of vemurafenib in 2011, and of dabrafenib and trametinib in 2013 in the USA.

\section{Vemurafenib}

The intracranial activity of vemurafenib was suggested by a pilot study conducted by Dummer et al. (Table 4) [67]. In this study, 24 patients with active BMs received vemurafenib at $960 \mathrm{mg}$ orally twice daily. At baseline, all patients were taking corticosteroid and had failed at least one prior therapeutic modality for BMs. Fifty percent of the patients had four or more brain lesions, and two-third exhibited CNS-related symptoms.

The primary end point was to evaluate the safety profile of vemurafenib in patients with active BMs. Secondary end points included intracranial and global overall response rates, PFS and OS. Confirmed intracranial PR was 16\% (95\% CI: 3.4-39.6\%). Intracranial disease stabilization was observed in $68 \%$ of patients (95\% CI: 43.4-87.4\%). The global overall response rate was $42 \%$ (95\% CI: $22.1-64.3 \%$ ). Median PFS and OS were 3.9 months (95\% CI: 3.0-5.5) and 5.3 months (95\% CI: 3.9-6.6), respectively. Patients' symptomatology improved with vemurafenib, as demonstrated by a reduction in pain score and corticosteroid requirement and improvement in performance status compared with baseline. The most common CNSrelated adverse events were grade 1 or 2 seizures (25\%) and headache (13\%).

The preliminary results of a Phase II study evaluating vemurafenib in 146 patients with $B R A F$-mutated advanced melanoma and active BMs were presented at the Society for 
Melanoma Research meeting in November 2013 [68]. Ninety patients with untreated BMs were assigned to cohort 1 , whereas 56 others with previously treated BMs were in cohort 2. The median number of BMs was 3.0 (range: 1-30) at enrollment. The investigator-assessed intracranial best overall response rate was $21 \%$ in cohort 1 . The primary end point, best overall response rate in the brain in cohort 1 as evaluated by central review committee, was not yet available. The median PFS and OS were 3.7 (range: 0.03-12.94) and 7.06 (range: $0.03-12.94)$ months in cohort 1 , respectively (Table 4).

\section{Dabrafenib}

BREAK-MB is a large Phase II study evaluating the safety and efficacy of dabrafenib at $150 \mathrm{mg}$ orally twice daily in 172 patients with $B R A F$ V600E- or V600K-mutated advanced melanoma and at least one asymptomatic BM measuring 5-40 $\mathrm{mm}$ in diameter (Table 4) [69]. Patients who had not received local therapy for BMs were assigned to cohort $\mathrm{A}$. Cohort $\mathrm{B}$ enrolled patients with progressive intracranial disease despite prior local therapy. Half of the patients had two to four brain lesions, and $81 \%$ of these patients had V600E mutation.

The primary end point of the study was the intracranial response in patients with V600Emutated melanoma. Secondary end points included intracranial response in patients with V600K mutation, overall response, duration of intracranial and overall response, PFS and OS. In patients with V600E mutation, intracranial response rates were $39.2 \%$ in cohort $\mathrm{A}$ and $30.8 \%$ in cohort $\mathrm{B}$, and median durations of intracranial tumor regression lasted 20.1 and 28.1 weeks in cohort $A$ and $B$, respectively. In those with V600K-mutated tumor, response rates in the brain were $6.7 \%$ in cohort $\mathrm{A}$ and $22.2 \%$ in cohort $\mathrm{B}$, and median durations of response in the brain were 12.4 weeks in cohort $A$ and 16.6 weeks in cohort B. Overall response rates, based on tumor response when both intra- and extracranial disease was assessed, were $37.8 \%$ (95\% CI: 26.8-49.9) and 30.8\% (95\%CI: 19.9-43.5) in patients with V600Emutated melanoma in cohort A and cohort B, respectively. Overall response rates were lower in those with V600K-mutated melanoma, 0\% (95\% CI: 0-21.8) in cohort A and 27.8\% (95\% CI: 9.7-53.5) in cohort B. The median OS was 31.4-33.1 weeks for those with V600E-positive tumor. The median OS was shorter for those with V600K-positive melanoma, ranging 16.3-21.9 weeks. The safety profile of dabrafenib in this study was similar to previous experience in patients without BMs. Intracranial hemorrhages occurred in ten patients, and one was treatment related.

\section{Dual MAPK blockade}

Despite the striking clinical benefit in patients with $B R A F$-mutated advanced melanoma, duration of response to MAPK pathway-targeted therapy is relatively short, implicating rapid emergence of drug resistance. Since MAPK reactivation is a common theme of tumor resistance mechanisms [86,87], dual MAPK pathway blockade with combined BRAF and MEK inhibitors has been shown to be advantageous in deterring resistance and limiting the development of squamoproliferative skin lesions mediated by BRAF inhibitor-induced paradoxical MAPK pathway activation in $B R A F$ wild-type cells [88,89].

Targeted therapy for melanoma BMs has also shifted toward dual MAPK blockade. COMBI-MB, an open-label, Phase II study evaluating dabrafenib-trametinib combination in patients with $B R A F \mathrm{~V} 600$-mutant melanoma and active $\mathrm{BMs}$, has started enrolling patients (NCT02039947).

\section{Conclusion}

In the past decade, more effective pharmacotherapies are leading to better systemic disease control in patients with advanced melanoma. Unfortunately, disease relapses in the brain remain a major obstacle to cure. The challenges for the oncology community are formidable, and great need exists to understand the mechanisms of BMs at the molecular level to develop innovative therapeutic strategies.

Advances in imaging, surgical and radiation techniques have improved the safety and effectiveness of local therapies, prolonging survival while preserving neurocognitive function for patients with melanoma BMs. Furthermore, median OS achieved with targeted and immunologic agents in patients with intracranial disease appeared favorable when compared with historical controls. This finding suggests that such novel pharmacotherapies produce meaningful intracranial response, expanding the systemic treatment options for patients with melanoma BMs. The clinical benefit of 
these pharmacotherapies becomes particularly important when intracranial disease burden precludes options of surgery or radiosurgery. Considerable effort in multidisciplinary approach is ongoing to improve outcomes for melanoma patients with BMs, a major challenge in the management of advanced melanoma.

\section{Future perspective}

Built upon the advances over the last decade, multidisciplinary efforts persevere to improve survival outcome and quality of life for patients with melanoma BMs. Real-time intraoperative imaging techniques able to identify cancer cells from normal brain tissues will ensure safer and more complete tumor resection. Similarly, innovative technologies are being evaluated to maximize radiotherapy dose at tumor site while minimizing exposure to other areas of the brain, especially the portion governing neurocognitive function. New evidence refuting the absolute impenetrable and immuno-privileged nature of the CNS environment lay platform for prospective clinical evaluation of novel immunotherapeutic and targeted agents in patients with active BMs. Meanwhile, scientific research continues tackling the biology of brain-tropic melanoma cells and their complex interactions with the cerebral microenvironment to guide drug development for the prevention and treatment of melanoma BMs.

Financial \& competing interests disclosure W-J Hwu receives grant and research funding from BristolMyers Squibb, Merck, GlaxoSmithKline, Medimmune and serves as an advisor on Merck Melanoma US Advisory Board. The authors have no other relevant affiliations or financialinvolvement with any organization or entity with a financial interest in or financial conflict with the subject matter or materials discussed in the manuscript apart from those disclosed.

No writing assistance was utilized in the production of this manuscript.

\section{References}

Papers of special note have been highlighted as: - of interest; $\bullet$ of considerable interest

1 Amer MH, Al-Sarraf M, Baker LH et al. Malignant melanoma and central nervous system metastases - incidence, diagnosis, treatment and survival. Cancer 42, 660-668 (1978).

2 Davies MA, Liu P, McIntyre S et al. Prognostic factors for survival in melanoma patients with brain metastases. Cancer 117(8), 1687-1696 (2011).

3 Raizer JJ, Hwu WJ, Panageas KS et al. Brain and leptomeningeal metastases from cutaneous melanoma: survival outcomes based on clinical features. Neuro Oncol. 10 (2), 199-207 (2008).

4 Sampson JH, Carter JH Jr, Friedman AH, Seigler HF. Demographics, prognosis, and therapy in 702 patients with brain metastases from malignant melanoma. J. Neurosurg. 88(1), 11-20 (1998).

5 Zakrzewski J, Geraghty LN, Rose AE et al. Clinical variables and primary tumor characteristics predictive of the development of melanoma brain metastases and post-brain metastases survival. Cancer 117(8), 1711-1720 (2011).

6 Bedikian AY, Wei C, Detry M et al. Predictive factors for the development of brain metastasis in advanced unresectable metastatic melanoma. Am. J. Clin. Oncol. 34, 603-610 (2011).
7 Qian M, Ma MW, Fleming NH et al. Clinicopathological characteristics at primary melanoma diagnosis as risk factors for brain metastasis. Melanoma Res. 23, 461-467 (2013).

8 Jakob JA, Bassett R, Ng CS et al. NRAS mutation status is an independent prognostic factor in metastatic melanoma. Cancer 118(16), 4014-4023 (2012).

9 Bucheit AD, Chen G, Siroy A et al. Complete loss of PTEN protein expression correlates with shorter time to brain metastasis and survival in stage IIIB/C melanoma patients with BRAFV600 mutations. Clin. Cancer Res. 20(21), 5527-5536 (2014).

10 Othus M, Moon J, Margolin K. Melanoma’s deadly march to the brain. Cancer 117(8), 1560-1563 (2011).

11 Staudt M, Lasithiotakis K, Leiter U et al. Determinants of survival in patients with brain metastases from cutaneous melanoma. Br. J. Cancer 102(8), 1213-1218 (2010).

12 Lonser RR, Song DK, Klapper J et al. Surgical management of melanoma brain metastases in patients treated with immunotherapy. J. Neurosurg. 115(1), 30-36 (2011).

13 Liew DN, Kano H, Kondziolka D et al. Outcome predictors of Gamma Knife surgery for melanoma brain metastases. Clinical article. J. Neurosurg. 114(3), 769-779 (2011).
14 Gaspar L, Scott C, Rotman M et al. Recursive partitioning analysis (RPA) of prognostic factors in three Radiation Therapy Oncology Group (RTOG) brain metastases trials. Int. J. Radiat. Oncol. Biol. Phys. 37(4), 745-751 (1997).

- The Radiation Therapy Oncology Group introduced the recursive partitioning analysis classification system, a prognostic index to predict outcome for patients with brain metastases (BMs) based on pretreatment variables.

15 Gaspar L, Scott C, Murray K et al. Validation of the RTOG recursive partitioning analysis (RPA) classification for brain metastases. Int J. Radiat. Oncol. Biol. Phys. 47, 1001-1006 (2000).

16 Sperduto PW, Berkey B, Gaspar LE et al. A new prognostic index and comparison to three other indices for patients with brain metastases: an analysis of 1960 patients in the RTOG database. Int. J. Radiat. Oncol. Biol. Phys. 70, 510-514 (2008).

17 Sperduto PW, Kased N, Roberge D et al. Summary report on the graded prognostic assessment: an accurate and facile diagnosisspecific tool to estimate survival for patients with brain metastases. J. Clin. Oncol. 30(4), 419-425 (2012).

- Established disease-specific criteria for prognosis assessment for patients with BMs. 
18 Patchell RA, Tibbs PA, Walsh JW et al. A randomized trial of surgery in the treatment of single metastases to the brain. $N$. Engl. J. Med. 322 (8), 494-500 (1990).

- Showed a survival benefit of surgical resection plus whole brain radiation therapy (WBRT) compared with WBRT alone in patients with solitary BMs.

19 Vecht CJ, Haaxma-Reiche H, Noordijk EM et al. Treatment of single brain metastasis: radiotherapy alone or combined with neurosurgery? Ann. Neurol. 33(6), 583-590 (1993).

-• Showed a survival benefit of surgical resection plus WBRT compared with WBRT alone in patients with solitary BMs.

20 Mintz AH, Kestle J, Rathbone MP et al. A randomized trial to assess the efficacy of surgery in addition to radiotherapy in patients with a single cerebral metastasis. Cancer 78 (7), 1470-1476 (1996).

21 Bindal RK, Sawaya R, Leavens ME, Lee JJ. Surgical treatment of multiple brain metastases. J. Neurosurg. 79(2), 210-216 (1993).

-• Suggested complete resection of multiple BMs may produce survival benefit comparable to complete resection of solitary BMs.

22 Konstadoulakis MM, Messaris E, Zografos G, Androulakis G, Karakousis C. Prognostic factors in malignant melanoma patients with solitary or multiple brain metastases. Is there a role for surgery? J Neurosurg. Sci. 44(4), 211-218; discussion 219 (2000).

- Evaluated the role of neurosurgery in patients with single or multiple melanoma BMs.

23 Doss LL, Memula N. The radioresponsiveness of melanoma. Int. J. Radiat. Oncol. Biol. Phys. 8(7), 1131-1134 (2000).

24 Brown PD, Brown CA, Pollock BE, Gorman DA, Foote RL. Stereotactic radiosurgery for patients with "radioresistant" brain metastases. Neurosurgery 51(3), 656-665 (2000).

- Evaluated the efficacy and safety of WBRT in patients with BMs.

25 Aoyama H, Shirato H, Tago M et al. Stereotactic radiosurgery plus whole brain radiation therapy vs stereotactic radiosurgery alone for the treatment of brain metastases: a randomized control trial. JAMA 295(21), 2483-2491 (2006).

26 Kocher M, Soffietti R, Abacioglu U et al. Adjuvant whole-brain radiotherapy versus observation after radiosurgery or surgical resection of one to three cerebral metastases: results of the EORTC 22952-26001 study. J. Clin. Oncol. 29(2), 134-141 (2011).

27 Fogarty G, Morton RL, Vardy J et al. Whole brain radiotherapy after local treatment of brain metastases in melanoma patients - a randomised Phase III trial. BMC Cancer 11, 142 (2011).

28 Hauswald H, Dittmar JO, Habermehl D et al. Efficacy and toxicity of whole brain radiotherapy in patients with multiple cerebral metastases from malignant melanoma. Radiat. Oncol. 7, 130 (2012).

- Evaluated the efficacy and safety of WBRT in patients with melanoma BMs.

29 Rades D, Heisterkamp C, Huttenlocher S et al. Dose escalation of whole-brain radiotherapy for brain metastases from melanoma. Int. J. Radiat. Oncol. Biol. Phys. 77(2), 537-541 (2010).

- Investigated the efficacy and safety of dose escalation of WBRT in patients with melanoma BMs.

30 Ellerhorst J, Strom E, Nardone E, McCutcheon I. Whole brain irradiation for patients with metastatic melanoma: a review of 87 cases. Int. J. Radiat. Oncol. Biol. Phys. 49(1), 93-97 (2001).

31 Dyer MA, Arvold ND, Chen YH et al. The role of whole brain radiation therapy in the management of melanoma brain metastases. Radiat. Oncol. 9, 143 (2014).

- Investigated the role of additional up-front WBRT in patients with melanoma BMs treated with stereotactic radiosurgery.

32 Schild SE, Behl D, Markovic SN et al. Brain metastases from melanoma: is there a role for concurrent temozolomide in addition to whole brain radiation therapy? Am. J. Clin. Oncol. 33(6), 633-636 (2010).

33 Devito N, Yu M, Chen R, Pan E. Retrospective study of patients with brain metastases from melanoma receiving concurrent whole-brain radiation and temozolomide. Anticancer Res. 31(12), 4537-4543 (2010).

34 Margolin K, Atkins B, Thompson A et al. Temozolomide and whole brain irradiation in melanoma metastatic to the brain: a Phase II trial of the Cytokine Working Group. J. Cancer Res. Clin. Oncol. 128(4), 214-218 (2002).

35 Mornex F, Thomas L, Mohr P et al. A prospective randomized multicenter Phase III trial of fotemustine plus whole brain irradiation versus fotemustine alone in cerebral metastases of malignant melanoma. Melanoma Res. 13, 97-103 (2003).
36 Gerber NK, Young RJ, Barker CA et al. Ipilimumab and whole brain radiation therapy for melanoma brain metastases. J. Neurooncol. 121(1), 159-165 (2015).

37 Dziggel L, Segedin B, Podvrsnik NH, Oblak I, Schild SE, Rades D. A survival score for patients with brain metastases from less radiosensitive tumors treated with wholebrain radiotherapy alone. Strablenther. Onkol. 190(1), 54-58 (2014).

38 Huttenlocher S, Sehmisch L, Schild SE, Blank O, Hornung D, Rades D. Identifying melanoma patients with $1-3$ brain metastases who may benefit from whole-brain irradiation in addition to radiosurgery. Anticancer Res. 34(10), 5589-5592 (2014).

39 Kondziolka D, Patel A, Lunsford LD, Kassam A, Flickinger JC. Stereotactic radiosurgery plus whole brain radiotherapy versus radiotherapy alone for patients with multiple brain metastases. Int. J. Radiat. Oncol. Biol. Phys. 45(2), 427-434 (1999).

40 Andrews DW, Scott CB, Sperduto PW et al. Whole brain radiation therapy with or without stereotactic radiosurgery boost for patients with one to three brain metastases: Phase III results of the RTOG 9508 randomised trial. Lancet 363(9422), 1665-1672 (2004).

41 Grob JJ, Regis J, Laurans R et al. Radiosurgery without whole brain radiotherapy in melanoma brain metastases. Club de Cancerologie Cutanee. Eur. J. Cancer 34(8), 1187-1192 (1998).

- Evaluated the efficacy and safety of gamma knife in patients with melanoma BMs.

42 Mori Y, Kondziolka D, Flickinger JC, Kirkwood JM, Agarwala S, Lunsford LD. Stereotactic radiosurgery for cerebral metastatic melanoma: factors affecting local disease control and survival. Int. J. Radiat. Oncol. Biol. Phys. 42(3), 581-589 (1998).

- Evaluated the efficacy and safety of gamma knife in patients with melanoma BMs.

43 Herfarth KK, Izwekowa O, Thilmann C et al. Linac-based radiosurgery of cerebral melanoma metastases. Analysis of 122 metastases treated in 64 patients. Strablenther. Onkol. 179(6), 366-371 (2003).

- Evaluated the efficacy and safety of linac-based stereotactic radiosurgery in patients with melanoma BMs.

44 Selek U, Chang EL, Hassenbusch SJ 3rd et al. Stereotactic radiosurgical treatment in 103 patients for 153 cerebral melanoma metastases. Int. J. Radiat. Oncol. Biol. Phys. 59(4), 1097-1106 (2004). 
- Evaluated the efficacy of linac-based stereotactic radiosurgery in a large population of patients with melanoma BMs.

45 Chang EL, Selek U, Hassenbusch SJ 3rd et al. Outcome variation among "radioresistant" brain metastases treated with stereotactic radiosurgery. Neurosurgery 56(5), 936-945 (2005).

- Assessed the variation in outcome of patients with BMs from melanoma, sarcoma or renal cell carcinoma treated with linac-based stereotactic radiosurgery.

46 Mathieu D, Kondziolka D, Copper PB et al. Gamma knife radiosurgery in the management of malignant melanoma brain metastases. Neurosurgery 60(3), 471-482 (2007).

- Evaluated the efficacy of gamma knife radiosurgery in a large population of patients with melanoma BMs.

47 Chang EL, Wefel JS, Hess KR et al. Neurocognition in patients with brain metastases treated with radiosurgery or radiosurgery with whole-brain irradiation: a randomized controlled trial. Lancet Oncol. 10(11), 1037-1044 (2009).

- Demonstrated the negative impact of radiosurgery plus WBRT on neurocognitive function compared with radiosurgery alone in patients with BMs.

48 Onodera S, Aoyama H, Tha KK et al. The value of 4-month neurocognitive function as an endpoint in brain metastases trials. J. Neurooncol. 120 (2), 311-319 (2014).

49 Bhatnagar AK, Flickinger JC, Kondziolka D, Lunsford LD. Stereotactic radiosurgery for four or more intracranial metastases. Int. J. Radiat. Oncol. Biol. Phys. 64(3), 898-903 (2006).

50 Redmond AJ, Diluna ML, Hebert R et al. Gamma Knife surgery for the treatment of melanoma metastases: the effect of intratumoral hemorrhage on survival. J. Neurosurgery 109(Suppl.), 99-105 (2008).

51 Likhacheva A, Pinnix CC, Parikh N et al. Validation of recursive partitioning analysis and diagnosis-specific graded prognostic assessment in patients treated initially with radiosurgery alone. J. Neurosurgery 117(Suppl.), 38-44 (2012).

52 Yamamoto M, Kawabe T, Sato Y et al. Stereotactic radiosurgery for patients with multiple brain metastases: a case-matched study comparing treatment results for patients with 2-9 versus 10 or more tumors. J. Neurosurgery 121(Suppl. 2), 16-25 (2014).
53 Clarke JW, Register S, McGregor JM et al. Stereotactic radiosurgery with or without whole brain radiotherapy for patients with a single radioresistant brain metastasis. Am. J. Clin. Oncol. 33(1), 70-74 (2010).

54 Gerson SL. Alkylating agents. In: Cancer Chemotherapy and Biotherapy: Principles and Practice (5th Edition). Chabner BA, Longo DL (Eds). Lippincott Williams and Wilkins, PA, USA, 268-309 (2011).

55 Proleukin $^{\circledR}$ (Aldesleukin), prescribing information. Prometheus Laboratories, Inc., CA, USA (2012). www.proleukin.com/assets/proleukin.pdf

56 Agarwala SS. Current systemic therapy for metastatic melanoma. Expert Rev. Anticancer Ther. 9, 587-595 (2009).

57 Ostermann S, Csajka C, Buclin T et al. Plasma and cerebrospinal fluid population pharmacokinetics of temozolomide in malignant glioma patients. Clin. Cancer Res. 10, 3728-3736 (2004).

58 Agarwala SS, Kirkwood JM, Gore M et al. Temozolomide for the treatment of brain metastases associated with metastatic melanoma: a Phase II study. J. Clin. Oncol. 22, 2101-2107 (2004).

- Evaluated the efficacy and safety of temozolomide in patients with melanoma BMs.

59 Hwu WJ, Lis E, Menell JH et al. Temozolomide plus thalidomide in patients with brain metastases from melanoma: a Phase II study. Cancer 103, 2590-2597 (2005).

60 Krown SE, Niedzwiecki D, Hwu WJ, Hodgson L, Houghton AN, Haluska FG. Phase II study of temozolomide and thalidomide in patients with metastatic melanoma in the brain - high rate of thromboembolic events (CALGB 500102). Cancer 107, 1883-1890 (2006).

61 Jacquillat CI, Kayat D, Banzet P et al. Chemotherapy by fotemustine in cerebral metastases of disseminated malignant melanoma. Cancer Chemother. Pharmacol. 25, 263-266 (1990).

- Evaluated the efficacy and safety of fotemustine in patients with melanoma BMs.

62 Jacquillat CI, Kayat D, Banzet P et al. Final report of the French multicenter Phase II study of the nitrosourea fotemustine in 153 evaluable patients with disseminated malignant melanoma including patients with cerebral metastases. Cancer 66, 1873-1878 (1990).

63 Calabresi E, Aapro M, Becquart D et al. Multicenter Phase II trial of the single agent fotemustine in patients with advanced malignant melanoma. Ann. Oncol. 2, 377-378 (1991).

64 Avril MF, Aamdal S, Grob JJ et al. Fotemustine compared with dacarbazine in patients with disseminated malignant melanoma: a Phase III study. J. Clin. Oncol. 22, 1118-1125 (2004).

- Compared the efficacy and safety of fotemustine with dacarbazine in patients with disseminated malignant melanoma including those with BMs.

65 Margolin K, Ernstoff MS, Hamid O et al. Ipilimumab in patients with melanoma and brain metastases: an open-label, Phase 2 trial. Lancet Oncol. 13, 459-465 (2012).

- Evaluated the efficacy and safety of ipilimumab in patients with melanoma BMs.

66 Queirolo P, Spagnolo F, Ascierto PA et al. Efficacy and safety of ipilimumab in patients with advanced melanoma and brain metastases. J. Neurooncol. 118(1), 109-116 (2014).

67 Dummer R, Goldinger SM, Turtschi CP et al. Vemurafenib in patients with $B R A F^{V 600}$ mutation-positive melanoma patients with symptomatic brain metastases: final results of an open-label pilot study. Eur. J. Cancer 50, 611-621 (2014).

- Evaluated the efficacy and safety of vemurafenib in patients with melanoma BMs.

68 Kefford RF, Maio M, Arance A et al. Vemurafenib in metastatic melanoma patients with brain metastases: an open-label, single-arm, Phase 2, multicenter study. Pigment Cell Melanoma Res. 26, 965 (2013).

- Evaluated the efficacy and safety of vemurafenib in a large population of patients with melanoma BMs.

69 Long GV, Trefzer U, Davies MA et al. Dabrafenib in patients with Val600Glu or Val600Lys BRAF-mutant melanoma metastatic to the brain (BREAK-MB): a multicenter, open-label, Phase 2 trial. Lancet Oncol. 13, 1087-1095 (2012).

- Evaluated the efficacy and safety of dabrafenib in a large population of patients with melanoma BMs.

70 Stevens A, Kloter I, Roggendorf W. Inflammatory infiltrates and natural killer cell presence in human brain tumors. Cancer 61, 738-743 (1988).

71 Prins RM, Shu CJ, Radu CG et al. Antitumor activity and trafficking of self, tumor-specific $T$ cells against tumors located 
in the brain. Cancer Immunol. Immunother. 57(9), 1279-1289 (2008).

72 Gerstner ER, Fine RL. Increased permeability of the blood-brain barrier to chemotherapy in metastatic brain tumors: establishing a treatment paradigm. J. Clin. Oncol. 25(16), 2306-2312 (2007).

73 Di Giacomo AM, Ascierto PA, Pilla L et al. Ipilimumab and fotemustine in patients with advanced melanoma (NIBIT-M1): an open-label, single-arm, Phase 2 trial. Lancet Oncol. 13, 879-86 (2012).

74 Ipilimumab and Whole-Brain Radiation Therapy or Stereotactic Radiosurgery in Treating Patients With Melanoma With Brain Metastases. https://clinicaltrials.gov

75 A Study of Fotemustine(FTM) Vs FTM and Ipilimumab (IPI) or IPI and Nivolumab in Melanoma Brain Metastasis (NIBIT-M2). https://clinicaltrials.gov

76 A Multi-Center Phase 2 Open-Label Study to Evaluate Safety and Efficacy in Subjects With Melanoma Metastatic to the Brain Treated With Nivolumab in Combination With Ipilimumab Followed by Nivolumab Monotherapy (CheckMate 204). https://clinicaltrials.gov

77 Flies DB, Sandler BJ, Sznol M, Chen L. Blockade of the B7-H1/PD-1 pathway for cancer immunotherapy. Yale J. Biol. Med. 84, 409-421 (2011).

78 Sznol M, Chen L. Antagonist antibodies to PD-1 and B7-H1 (PD-L1) in the treatment of advanced human cancer. Clin. Cancer Res. 19, 1021-1034 (2013).

79 Topalian SL, Sznol M, McDermott DF et al. Survival, durable tumor remission, and long-term safety in patients with advanced melanoma receiving nivolumab. J. Clin. Oncol. 32, 1020-1030 (2014).

80 Ribas A, Hodi FS, Kefford R et al. Efficacy and safety of the anti-PD-1 monoclonal antibody pembrolizumab (MK-3475) in 411 patients with metastatic melanoma. Presented at: 2014 Annual Meeting of the American Society of Clinical Oncology. Chicago, IL, USA, 30 May-3 June 2015.

81 Lipson EJ, Sharfman WH, Drake CG et al. Durable cancer regression off-treatment and effective reinduction therapy with an anti-PD-1 antibody. Clin. Cancer Res. 19(2), 462-468 (2013).

82 MK-3475 in Melanoma and NSCLC Patients With Brain Metastases. https://clinicaltrials.gov

83 Wolchok JD, Kluger H, Callahan MK et al. Nivolumab plus ipilimumab in advanced melanoma. N. Engl. J. Med. 369, 122-133 (2013).
84 Davies H, Bignell GR, Cox C et al. Mutations of the BRAF gene in human cancer. Nature 417, 949-954 (2002).

85 Davies MA, Gershenwald JE. Targeted therapy for melanoma - a primer. Surg. Oncol. Clin. N. Am. 20(1), 165-180 (2011).

86 Noor R, Trinh VA, Kim KB, Hwu WJ. BRAF-targeted therapy for metastatic melanoma: rationale, clinical activity and safety. Clin. Invest. 1, 1127-1139 (2011).

87 Sullivan RJ, Flaherty KT. Resistance to BRAF-targeted therapy on melanoma. Eur. J. Cancer 49, 1297-1304 (2013).

88 Robert C, Karaszewska B, Schachter J et al. Improved overall survival in melanoma with combined dabrafenib and trametinib. N. Engl. J. Med. 372(1), 30-39 (2014).

89 Larkin J, Ascierto PA, Dreno B et al. Combined vemurafenib and cobimetinib in BRAF-mutated melanoma. N. Engl. J. Med. 371(20), 1867-1876 (2014).

90 Study to Evaluate Treatment of Dabrafenib Plus Trametinib in Subjects With BRAF Mutation-Positive Melanoma That Has Metastasized to the Brain. https://clinicaltrials.gov 